\title{
Composição mineral de uma linhagem de Pleurotus ostreatus cultivada em resíduos madeireiros e agroindustriais da região amazônica
}

\author{
Mineral composition of Pleurotus ostreatus strain grown in wood and \\ agroindustrial residues from the Amazon region
}

\author{
Ceci SALES-CAMPOS ${ }^{1 *}$, Luiz Antônio de OLIVEIRA ${ }^{1}$, Lidia Medina ARAUJO1, \\ Maria de Jesus Coutinho VAREJÃO ${ }^{1}$, Meire Cristina Nogueira de ANDRADE $^{1}$
}

\section{Resumo}

Os cogumelos do gênero Pleurotus são cultivados em diversos substratos lignocelulósicos, dada a atividade decompositora desses organismos proveniente de seu metabolismo enzimático. O presente estudo teve como objetivo analisar a composição mineral de Pleurotus ostreatus e dos substratos de cultivo preparados à base de resíduos madeireiros e agroindustriais da região amazônica. Foram analisados macro ( $\mathrm{P}$, K, $\mathrm{Ca}$ e $\mathrm{Mg}$ ) e micronutrientes ( $\mathrm{Fe}, \mathrm{Zn}, \mathrm{Cu}, \mathrm{Mn}$ e $\mathrm{Na}$ ) dos cogumelos e dos substratos. Os substratos foram formulados a partir da serragem de Simarouba amara Aubl. (marupá), Ochroma piramidale Cav. ex. Lam. (pau de balsa) e de bagaços de Bactris gasipaes Kunth (pupunheira) e de Saccharum officinarum (cana-de-açúcar). As amostras foram solubilizadas mediante digestão ácida (nítrico-peridrol). Os elementos Ca, $\mathrm{Mg}, \mathrm{Fe}, \mathrm{Cu}, \mathrm{Zn}$ e Mn foram determinados por espectrometria de absorção atômica; o $\mathrm{Na}$ e K, por emissão atômica e o P, por colorimetria. A composição mineral do cogumelo variou com o substrato de cultivo. Os diferentes substratos possibilitaram a produção de um cogumelo rico em $\mathrm{K}, \mathrm{P}, \mathrm{Mg}$ e Fe, essenciais à nutrição e à saúde humana. O potássio foi o mineral de maior teor no cogumelo em todos os substratos testados (36,83-42,18 g. $\left.\mathrm{kg}^{-1}\right)$, seguido de fósforo (6,95-10,60 g.kg-1) e do magnésio $\left(1,57-2,50 \mathrm{~g}^{-\mathrm{kg}^{-1}}\right)$.

Palavras-chave: Pleurotus; cogumelo; substrato; composição mineral.

\begin{abstract}
Mushrooms belonging to the Pleurotus gender are grown in several lignocellulosic substrates due to the decomposing activity of these organisms that result from their enzymatic metabolism. The objective of the present study was to analyze the mineral composition of Pleurotus ostreatus and the cultivation substrates prepared with wood and agroindustrial residues from the Amazon region. Macro (P, K, $\mathrm{Ca}$ and $\mathrm{Mg}$ ) and micronutrients ( $\mathrm{Fe}, \mathrm{Zn}, \mathrm{Cu}, \mathrm{Mn}$ and $\mathrm{Na}$ ) of mushroom and substrates were analyzed. Substrates were formulated from Simarouba amara Aubl. and Ochroma piramidale Cav. ex. Lam. sawdust and crushed Bactris gasipaes Kunth, and Saccharum officinarum (sugar cane). The samples were solubilized by acid digestion (nítric-peridrol). The elements $\mathrm{Ca}, \mathrm{Mg}, \mathrm{Fe}, \mathrm{Cu}, \mathrm{Zn}$ and $\mathrm{Mn}$ were determined by atomic absorption spectrometry; $\mathrm{Na}$ and $\mathrm{K}$, by atomic emission, and $\mathrm{P}$ by colorimetry. The mineral composition of the mushroom varied with the substrate. Different substrates made possible the production of a mushroom rich in $\mathrm{K}, \mathrm{P}, \mathrm{Mg}$, and Fe, essential to human nutrition and health. Potassium was the mineral with the highest content in the mushroom for all substrates tested $\left(36.83-42.18 \mathrm{~g} . \mathrm{kg}^{-1}\right)$ followed by phosphorus (6.95-10.60 g. $\left.\mathrm{kg}^{-1}\right)$ and magnesium (1.57-2.50 g. kg $\left.{ }^{-1}\right)$.
\end{abstract}

Keywords: Pleurotus; mushroom; substrate; mineral composition.

\section{Introdução}

O cultivo de cogumelos comestíveis é uma atividade de grande importância econômica, principalmente a produção de espécies dos gêneros Agaricus, Pleurotus e Lentinula (GUZMÁN et al., 1993).

O aumento da produção mundial de cogumelos comestíveis, em especial do gênero Pleurotus, se deu em particular pela capacidade que as espécies têm de colonização e produção em uma variedade de resíduos, dentre eles a serragem e os oriundos da agroindústria, uma característica que viabilizou economicamente a produção (EIRA et al., 1997). Tais características os tornam importantes não só quanto ao seu papel na produção mas também do ponto de vista nutricional.
O tipo de substrato, as condições ambientais e a espécie de fungo utilizada no cultivo influenciam significativamente na composição química dos cogumelos, podendo ocorrer variações, principalmente, em relação a minerais e à qualidade proteica deles (CRISAN; SANDS, 1978; ANDRADE, 2007).

Alguns estudos acerca da composição mineral dos cogumelos cultivados têm sido feito, entre eles, Gbolagade et al. (2006), Strmisková, Strmiska e Dubravicky (1992), Vetter (1994), Sturion e Ranzani (2000). No entanto, adaptação de linhagens de Pleurotus spp. a novos resíduos requer mais do que conhecimentos inerentes ao processo de cultivo, necessita também de conhecimentos sobre a composição química do

Recebido para publicação em $7 / 2 / 2008$

Aceito para publicação em 14/11/2008 (003208)

Instituto Nacional de Pesquisas da Amazônia-INPA, Coordenação de Pesquisas em Produtos Florestais-CPPF, Av. André Araújo, 2936, Aleixo, CP 478, CEP 69060-001, Manaus - AM, Brasil,E-mail: ceci@inpa.gov.br

${ }^{*}$ A quem a correspondência deve ser enviada 
substrato e do cogumelo, sobretudo quando se trata de novas formulações com resíduos madeireiros e agroindustriais da Amazônia, onde esse tipo de estudo está sendo introduzido.

Assim, o presente estudo teve por fim analisar a composição mineral de cogumelos de $P$. ostreatus cultivados a partir de resíduos madeireiros e da agroindústria da Amazônia, visando contribuir com novos estudos acerca do cultivo de cogumelos, para futura aplicação no cultivo de fungos comestíveis na região.

\section{Material e métodos}

\subsection{Amostras analisadas}

Os substratos e cogumelos empregados no estudo foram nomeados da seguinte forma:

a) Substrato inicial autoclavado (SIA), antes de ser submetido ao cultivo do fungo, composto pela mistura de serragem ou bagaço $(78 \%)+$ mistura de farelos [Os farelos utilizados na mistura (MFR) foram arroz e trigo na proporção 2:1 (g/g)] (MFR) $(18 \%)+$ carbonato de cálcio $(2 \%)$, cuja mistura foi umedecida com água destilada (70\%). Adotou-se a seguinte codificação: SIAMP - substrato inicial formulado a partir da serragem de marupá; SIAPB - da serragem de pau de balsa; SRPP - do resíduo do estipe da pupunheira; SIACN - do bagaço de cana-de-açúcar. Os substratos foram autoclavados a $121^{\circ} \mathrm{C}$ durante 1 hora.

b) Cogumelo cultivado nos respectivos substratos iniciais: SIAMP-COG; SIAPB-COG; SIAPP-COG; e SIACN-COG.

\subsection{Determinação de macro e micronutrientes}

As análises foram realizadas em triplicata, seguindo o mesmo procedimento utilizado para análise de solos e plantas descrito por Malavolta, Vitti e Oliveira (1989) e Malavolta (1992). As amostras foram desidratadas primeiramente ao ar livre e sequencialmente em estufa de circulação de ar forçada a $55 \pm 5{ }^{\circ} \mathrm{C}$. Em seguida, foram moídas em moinho de faca, tipo Willey, nas dependências da Coordenação de Pesquisas em Produtos Florestais do Instituto Nacional de Pesquisas da Amazônia - CPPF/INPA e encaminhadas para digestão e análises nos Laboratórios de Análise de Solos e Plantas do mesmo Instituto e da Central Analítica da Universidade Federal do Amazonas. Efetuou-se análise dos macronutrientes: cálcio, magnésio, fósforo, potássio; e dos micronutrientes: sódio, ferro, cobre, manganês e zinco.

As amostras foram pesadas em uma balança analítica $(0,5000 \mathrm{~g})$, digeridas com ácido nítrico-peridrol e solubilizadas. Os elementos foram analisados por espectrometria de absorção atômica, emissão atômica e colorimetria por UV-visível previamente calibradas com soluções padronizadas para cada elemento (AOAC, 1997).

O conteúdo de cálcio, magnésio, ferro, cobre, manganês e zinco foram determinados por espectrofotometria de absorção atômica. O fósforo foi determinado por colorimetria, sódio e potássio por emissão atômica. Os valores dos macronutrientes (Ca, P, Mg, K) foram calculados em g. $\mathrm{kg}^{-1}$ e os dos micros (Na, $\mathrm{Fe}, \mathrm{Cu}, \mathrm{Mn}$ e $\mathrm{Zn}$ ) em mg. $\mathrm{kg}^{-1}$.

\section{Resultados e discussão}

A tabela 1 apresenta os teores de macro e micronutrientes nos diferentes substratos de cultivo.

Os macronutrientes presentes nos substratos iniciais (Tabela 1) obedeceram à seguinte ordem decrescente para SIAPB e SIAPP: Ca, K, P e Mg, enquanto que para SIAMP e SIACN a ordem decrescente foi: $\mathrm{Ca}, \mathrm{P}, \mathrm{K}$ e Mg. Já os micros obtiveram a seguinte: $\mathrm{Na}, \mathrm{Fe}$, (exceto para SIAPP e SIACN, em que o Fe foi superior ao $\mathrm{Na}$ ), $\mathrm{Mn}, \mathrm{Zn}$ e $\mathrm{Cu}$. A presença destes minerais nos substratos testados confirma sua importância para o cultivo de cogumelos (KURTZMAN; ZADRAZIL, 1982; MOLENA, 1986; CHANG; MILES, 1989; MILES; CHANG, 1997).

$\mathrm{Na}$ Tabela 2 apresentam-se os resultados dos minerais presentes no cogumelo $P$. ostreatus cultivado nos diferentes substratos, caracterizando-o como fonte de minerais, os quais estão de acordo com os trabalhos de Chang e Miles (1989); Miles

Tabela 1. Composição mineral dos substratos iniciais autoclavados - SIA.

\begin{tabular}{|c|c|c|c|c|c|c|c|c|c|}
\hline \multirow[t]{3}{*}{ Substrato inicial autoclavado } & \multicolumn{4}{|c|}{ Macronutrientes } & \multicolumn{5}{|c|}{ Micronutrientes } \\
\hline & $\mathrm{Ca}$ & $\mathrm{Mg}$ & $\mathrm{P}$ & $\mathrm{K}$ & $\mathrm{Na}$ & $\mathrm{Fe}$ & $\mathrm{Zn}$ & $\mathrm{Mn}$ & $\mathrm{Cu}$ \\
\hline & \multicolumn{4}{|c|}{ g. $\mathrm{kg}^{-1}$} & \multicolumn{5}{|c|}{ mg.kg ${ }^{-1}$} \\
\hline SIAMP & 9,79 & 1,49 & 3,78 & 3,67 & 67,03 & 64,00 & 21,33 & 34,67 & 3,49 \\
\hline SIAPB & $(0,61)$ & $(0,12)$ & $(0,01)$ & $(0,32)$ & $(0,16)$ & $(1,53)$ & $(6,66)$ & $(1,00)$ & $(0,06)$ \\
\hline \multirow[t]{2}{*}{ SIAPP } & $(2,22)$ & $(0,11)$ & $(0,17)$ & $(0,44)$ & $(0,27)$ & $(1,15)$ & $(1,00)$ & $(0,00)$ & $(0,04)$ \\
\hline & 36,86 & 2,38 & 7,18 & 8,55 & 66,85 & 104,33 & 82,00 & 40,00 & 9,35 \\
\hline \multirow[t]{2}{*}{ SIACN } & $(0,02)$ & $(0,01)$ & $(0,01)$ & 0,03 & $(1,37)$ & $(0,58)$ & $(2,00)$ & $(1,53)$ & $(0,98)$ \\
\hline & 8,42 & 1,49 & 3,55 & 3,35 & 41,15 & 67,67 & 32,00 & 37,33 & 9,94 \\
\hline
\end{tabular}

SIAMP: substrato inicial formulado a partir da serragem de marupá; SIAPB: da serragem de pau de balsa; SIAPP: do estipe da pupunheira triturado; SIACN: do bagaço de cana. Números em negrito: média de três repetições por substrato em relação a cada mineral. Valores entre parênteses referem-se ao desvio padrão. 
e Chang (1997); Vetter (1990; 1994); Sturion e Ranzani (2000); Zhang e Fadel (2002); Bernás et al. (2006).

Os minerais presentes nos cogumelos são retirados do substrato por meio do micélio, durante o crescimento deste, e transferidos para o cogumelo durante o seu processo de formação (CHANG; MILES, 1989).

Os constituintes minerais dos cogumelos são basicamente os mesmos das plantas superiores (MOLENA, 1986). Assim como nestas, dentre todos os minerais analisados, o K apresenta-se como constituinte presente em maior concentração, seguido de $\mathrm{P}$ e $\mathrm{Mg}$, confirmando os dados da literatura (CHANG; LAU; CHO, 1981; CHANG; MILES, 1989; STRMISKOVÁ; STRMISKA; DUBRAVICKY, 1992; VETTER,1990; 1994; STURION, 1994; STURION; OETTERER, 1995; MILES; CHANG, 1997; STURION; RANZANI, 2000; WANG; SAKODA; SUZUKI, 2001; BERNÁS et al., 2006). O potássio variou de $36,83-42,18 \mathrm{~g} \cdot \mathrm{kg}^{-1}$, seguido de fósforo $\left(6,95-10,60 \mathrm{~g} \cdot \mathrm{kg}^{-1}\right)$, magnésio $\left(1,57-2,50 \mathrm{~g} \cdot \mathrm{kg}^{-1}\right)$ e cálcio $\left(0,34-0,60 \mathrm{~g} \cdot \mathrm{kg}^{-1}\right)$.

Os valores de potássio, fósforo e magnésio do cogumelo cultivado nos diversos resíduos (Tabela 2) são superiores aos apresentados por Vetter (1990) e também por Sturion (1994), em que a autora cultivou diversas linhagens de Pleurotus spp. em diferentes resíduos agrícolas. Os trabalhos de Chang et al. (1981), Vetter (1994) e o de Sturion e Ranzani (2000) relatam resultados de potássio e de magnésio analisados em diversas linhagens comerciais de Pleurotus spp. cultivados no Brasil.

Segundo Li e Chang (1982), apud Chang e Miles (1989), os teores de potássio, fósforo, cálcio, magnésio e sódio do cogumelo constituem cerca de 56 a $70 \%$ de seu conteúdo total de cinzas, sendo que o potássio contribui com aproximadamente $45 \%$, o que demonstra a riqueza desse mineral no cogumelo.

Potássio, fósforo e magnésio (este último, com exceção para o cogumelo cultivado no substrato oriundo de resíduo de pupunheira) estiveram presentes em maior quantidade no cogumelo do que no substrato inicial conforme comparação das Tabelas 1 e 2. O mesmo foi constatado por Bano e Rajarathnam (1988) apud Sturion (1994) para o fósforo, quando cultivaram diferentes espécies de Pleurotus spp. em palha de arroz. O conteúdo de fósforo e de potássio também foi maior no cogumelo em relação ao substrato de cultivo nos trabalhos de Zhang e Fadel (2002), quando cultivaram Pleurotus sajor-caju em palha de arroz e de trigo. Resultados semelhantes foram observados para magnésio e potássio no estudo de Sapata(2005), ou seja, estes nutrientes também foram maiores no cogumelo do que no substrato.

O teor de cálcio detectado no cogumelo, que aparece em menor concentração no atual estudo $\left(0,34\right.$ a 0,60 g. $\left.\mathrm{kg}^{-1}\right)$ (Tabela 2), está de acordo com os dados da literatura, como pode ser visto em todos os estudos reportados na tabela 3 , exceto para o cogumelo Pleurotus tuber-regium em que o conteúdo de cálcio é mais alto do que o de fósforo.

Entre os microminerais presentes no cogumelo cultivado nos diversos substratos analisados, o maior conteúdo apresentado foi para o sódio em todos os substratos testados, variando de 154-194,40 mg. $\mathrm{kg}^{-1}$, seguido de ferro (115,67-151 mg. $\left.\mathrm{kg}^{-1}\right)$, zinco (64,67-82 mg. $\left.\mathrm{kg}^{-1}\right)$, manganês (16-23 mg.kg $\left.{ }^{-1}\right)$ e cobre $(9,10-11,69$ $\left.\mathrm{mg} \cdot \mathrm{kg}^{-1}\right)$. Embora o sódio tenha tido o valor mais alto entre os microminerais, este ainda é considerado baixo, do ponto de vista de dieta humana, segundo Sturion e Ranzani (2000). Este fato, aliado à riqueza em potássio, faz deste cogumelo um alimento importante para pacientes com problemas de hipertensão.

Para micronutrientes em basidiomas, Strimisková et al. (1992) obtiveram a ordem decrescente em $\mathrm{mg} \mathrm{kg}^{-1}$ : sódio, ferro, zinco, cobre e manganês, os respectivos valores $(\mathrm{Na}=195,0>\mathrm{Fe}=90$, $\left.3>\mathrm{Zn}=67,6>\mathrm{Cu}=15,1>\mathrm{Mn}=7,4 \mathrm{mg} \cdot \mathrm{kg}^{-1}\right)$. A ordem é semelhante à do atual estudo, exceto para o manganês que foi superior ao cobre.

Houve uma variação da composição química do cogumelo de acordo com o substrato em que foi cultivado (Tabela 2). O mesmo foi relatado em estudos anteriores (CHANG; LAU; CHO, 1981; FASIDI; EKUERE, 1993; STURION, 1994; SILVA; COSTA; CLEMENTE, 1981).

Tabela 2. Composição mineral do cogumelo Pleurotus ostreatus cultivado nos diferentes substratos.

\begin{tabular}{|c|c|c|c|c|c|c|c|c|c|}
\hline \multirow[t]{3}{*}{ Cogumelo por substrato } & \multicolumn{4}{|c|}{ Macronutrientes } & \multicolumn{5}{|c|}{ Micronutrientes } \\
\hline & $\mathrm{Ca}$ & $\mathrm{Mg}$ & $\mathrm{P}$ & $\mathrm{K}$ & $\mathrm{Na}$ & $\mathrm{Fe}$ & $\mathrm{Zn}$ & $\mathrm{Mn}$ & $\mathrm{Cu}$ \\
\hline & \multicolumn{4}{|c|}{ g. $\mathrm{kg}^{-1}$} & \multicolumn{5}{|c|}{$\mathrm{mg} \cdot \mathrm{kg}^{-1}$} \\
\hline SIAMPCOG & 0,47 & 2,50 & 7,40 & 39,68 & 154,00 & 151,00 & 118,00 & 23,00 & 11,69 \\
\hline SIAPB COG & $(0,01)$ & $(0,02)$ & $(0,22)$ & $(1,01)$ & $(5,40)$ & $(1,15)$ & $(1,00)$ & $(0,58)$ & $(0,32)$ \\
\hline \multirow[t]{2}{*}{ SIAPP COG } & $(0,04)$ & $(0,01)$ & $(0,13)$ & $(0,43)$ & $(2,12)$ & $(1,15)$ & $(2,65)$ & $(0,00)$ & $(0,31)$ \\
\hline & 0,57 & 1,57 & 9,74 & 42,18 & 181,90 & 115,67 & 82,00 & 16,00 & 9,10 \\
\hline \multirow[t]{2}{*}{ SIACN COG } & $(0,03)$ & $(0,02)$ & $(0,28)$ & $(0,74)$ & $(8,20)$ & $(1,00)$ & $(1,00)$ & $(0,58)$ & $(0,27)$ \\
\hline & 0,60 & 2,12 & 6,95 & 41,52 & 194,40 & 123,00 & 96,00 & 20,67 & 10,39 \\
\hline
\end{tabular}

SIAMP-COG: cogumelo cultivado no substrato a partir da serragem de marupá; SIAPB-COG: da serragem de pau de balsa; SIAPP-COG: do estipe da pupunheira triturado; SIACN-

COG: do bagaço de cana. Números em negrito: média de três repetições por substrato em relação a cada mineral. Valores entre parênteses referem-se ao desvio padrão. 
Sales-Campos et al.

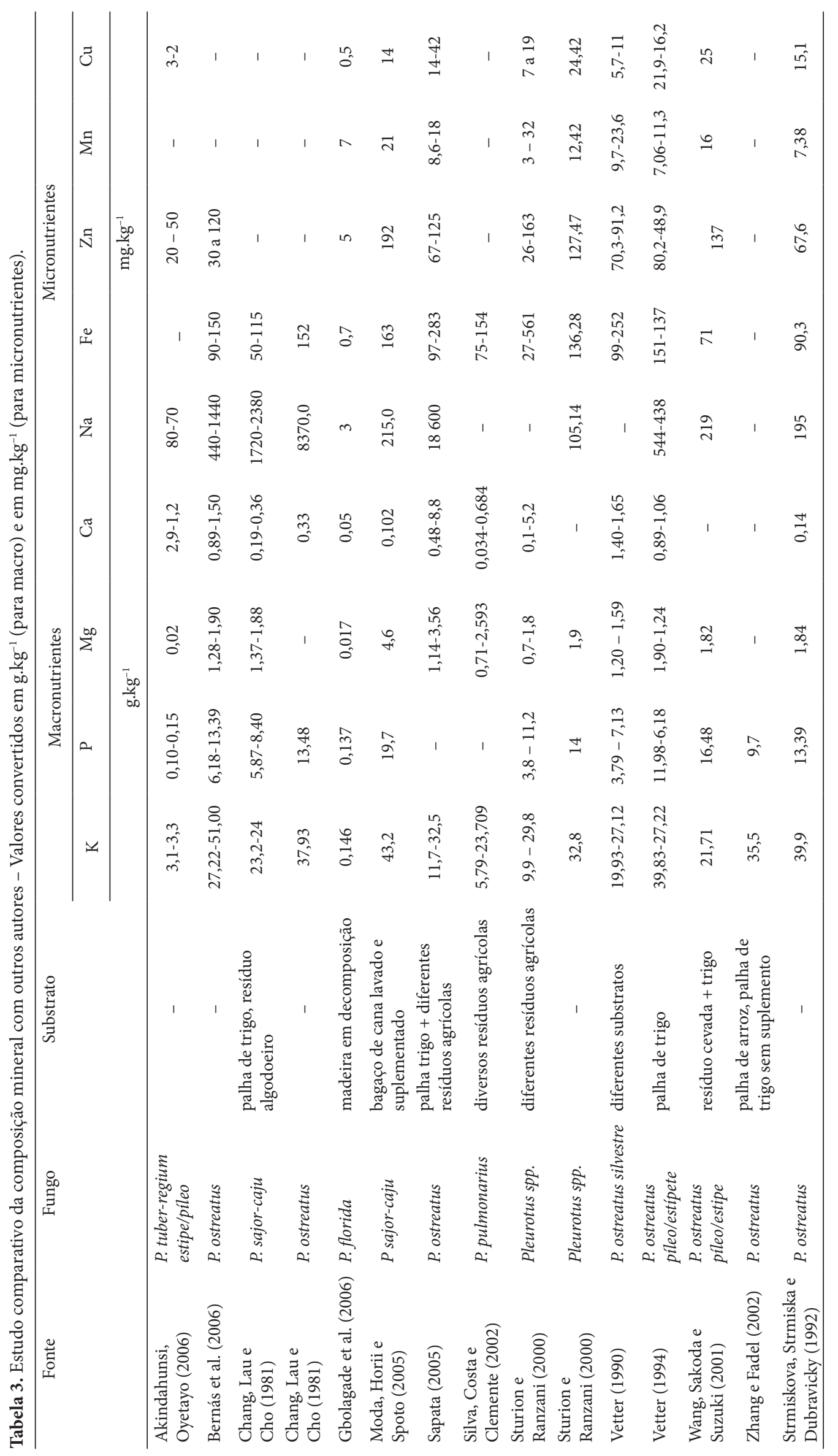




\section{Conclusões}

- A composição mineral do cogumelo variou com o substrato de cultivo;

- O potássio foi o mineral de maior conteúdo no cogumelo, em todos os substratos testados; e

- Os diferentes substratos utilizados no presente estudo possibilitaram a produção de um cogumelo rico em minerais: $\mathrm{K}, \mathrm{P}, \mathrm{Mg}$ e $\mathrm{Fe}$, essenciais à nutrição e saúde humana.

\section{Referências bibliográficas}

ASSOCIATION OF OFFICIAL ANALYTICAL CHEMISTS - AOAC. Official methods of analysis of AOAC International. $16 \mathrm{ed}$. Gaithersburg: AOAC International, 1997.

ANDRADE, M.C. N. Crescimento micelial, produção e características bromatológicas do shiitake em função de linhagens e de propriedades físicas e químicas de espécies e clones de eucalipto. Botucatu, 2007. 195 p. Tese (Doutorado em Agronomia) Universidade Estadual Paulista - UNESP.

AKINDAHUNSSI, A. A.; OYETAYO, F. L. Nutrient and antinutrient distribution of edible mushroom, Pleurotus tuber-regium (fries) Singer. Food Science and Technology, v. 39, n. 5, p. 548-553, 2006.

BERNÁS, B.; JAWORSKA, G.; LISIEWKA, Z. Edible Mushrooms as a souce of valuable nutritive constituintes. Acta Scientiarum Polonorum Technologia Alimentaria, v. 5, n. 1, p. 5-20, 2006.

CHANG, S. T.; LAU, O. W.; CHO, K. Y. The cultivation and nutritional value of Pleurotus sajor-caju. European Journal of Applied Microbiology and Biotechnology, v. 12, n. 1, p. 58-62, 1981.

CHANG, S. T.; MILES, P. G. Edible mushrooms and their cultivation. Florida: CRC Press, 1989.

CRISAN, E. V.; SANDS, A. A nutritional value. In: CHANG, S. T.; HAYES, W. A. (Eds). The biology and cultivation of edible mushroom. New York: Academic Press, 1978. p. 137-168.

EIRA, A. F. et al. 1997. Manual teórico/prático do cultivo de cogumelos comestíveis. Botucatu: FEPAF, 1997.

FASIDI, I.; EKUERE, V. U. Studies on Pleurotus tuber-regium (Fries) Singer: cultivation, proximate composition and mineral contents of sclerotia. Food Chemistry, v. 48, n. 3, p. 255-258, 1993.

GBOLAGADE, J. et al. Nutritive value of common wild edible mushrooms from nouthern Nigeria. Global Journal of Biotechnology and Biochemistry, v. 1, n. 1, p. 16-21, 2006.

GUZMÁN, G. et al. El cultivo de los hongos comestibles. México: Instituto Politécnico Nacional, 1993.

KURTZMAN, R. H.; ZADRAZIL, F. Physiological and taxonomic considerations for cultivation of Pleurotus mushrooms. In: CHANG, S. T.; QUIMIO, T. H. (Eds.). Tropical mushrooms: biological nature and cultivation methods. Hong Kong: The Chinese University Press, 1982. p. 299-348.

MALAVOLTA, E.; VITTI, G. C.; OLIVEIRA, S. A. Avaliação do estado nutricional das plantas: princípios e aplicações. Piracicaba: Editora Gráfica Nagy, 1989.

MALAVOLTA, E. ABC da análise de solos e folhas: amostragem, interpretação e sugestões de adubação. São Paulo: Agronômica Ceres, 1992.

MILES, P. G., CHANG, S. T. Mushroom biology: concise basics and current developments. Singapore: Word Scientific Publishing, 1997.

MODA, E. M.; HORII, J.; SPOTO, M. H. F. Edible mushroom Pleurotus sajor-caju production on washed and supplemented sugar cane bagasse. Scientia Agricola, v. 62, n. 2, p. 127-132, 2005.

MOLENA, O. O moderno cultivo de cogumelos. São Paulo: Nobel, 1986.

SAPATA, M. R. L. Valorização de resíduos agrícolas: produção de cogumelos do gênero Pleurotus. Oeiras: Instituto Nacional de Investigação Agrária e das Pescas; Estação Agronômica Nacional, 2005. 32 p. (Relatório final do projeto).

SILVA, S. O.; COSTA, S. M. G.; CLEMENTE, E. Chemical composition of Pleurotus pulmonarius (Fr.) Quél., substrates and residue after cultivation. Brazilian Archives of Biology and Technology, v. 45, n. 4, p. 531-535, 2002.

STRMISKOVÁ, G.; STRMISKA, F.; DUBRAVICKY, J. Mineral composition of oyester mushrooms. Nahrung, v. 36, n. 2, p. 210 212, 1992.

STURION, G. L. Utilização da folha da bananeira como substrato para o cultivo cogumelo (Pleurotus spp). Piracicaba, 1994. $147 \mathrm{p}$. Dissertação (Mestrado em Ciência e Tecnologia de Alimentos) Universidade do Estado de São Paulo - USP.

STURION, G. L.; RANZANI, M. R. T. C. Composição em minerais de cogumelos comestíveis cultivados no Brasil - Pleurotus spp. e outras espécies desidratadas. Archivos Latino Americanos de Nutricion, v. 50, n. 1, p. 102-103, 2000.

STURION, G. L.; OETTERER, M. Composição química de cogumelos comestíveis (Pleurotus spp.) originados em diferentes substratos. Ciência e Tecnologia de Alimentos, v. 15, n. 2, p. 189-193, 1995.

VETTER, J. Mineral element content of edible and poisonous macrofungi. Acta Alimentaria, v. 19, n. 1, p. 27-40, 1990.

VETTER, J. Mineral elements in the important cultivated mushrooms Agaricus bisporus and Pleurotus ostreatus. Food Chemistry, v. 50, n. 3, p. 277-279, 1994.

WANG, D.; SAKODA, A.; SUZUKI, M. Biological efficiency and nutritional value of Pleurotus ostreatus cultivated on spent beer grain. Bioresource Technology, v. 78, n. 3, p. 293-333, 2001.

ZHANG, R.; LI, X.; FADEL, J. G. Oyester mushroom cultivation with rice and wheat straw. Bioresource Technology, v. 82, n. 3, p. 277284, 2002. 